\title{
Hydrostatic Pressure Influences HIF-2 Alpha Expression in Chondrocytes
}

\author{
Hiroaki Inoue ${ }^{1}$, Yuji Arai ${ }^{1, *}$, Tsunao Kishida ${ }^{2}$, Ryu Terauchi ${ }^{1}$, Kuniaki Honjo ${ }^{1}$, \\ Shuji Nakagawa ${ }^{1}$, Shinji Tsuchida ${ }^{1}$, Tomohiro Matsuki ${ }^{1}$, Keiichirou Ueshima ${ }^{1}$, \\ Hiroyoshi Fujiwara ${ }^{1}$, Osam Mazda ${ }^{2}$ and Toshikazu Kubo ${ }^{1}$
}

1 Department of Orthopaedics, Graduate School of Medical Science, Kyoto Prefectural University of Medicine, Kawaramachi-Hirokoji, Kamigyo-ku, Kyoto 602-8566, Japan; E-Mails: hinoue@koto.kpu-m.ac.jp (H.I.); ryutel@mbox.kyoto-inet.or.jp (R.T.);

kuniakih@koto.kpu-m.ac.jp (K.H.); shushi@koto.kpu-m.ac.jp (S.N.); tuchi-kf@koto.kpu-m.ac.jp (S.T.); tmatsuki@koto.kpu-m.ac.jp (T.M.); ueshima@koto.kpu-m.ac.jp (K.U.); fjwr@koto.kpu-m.ac.jp (H.F.); tkubo@koto.kpu-m.ac.jp (T.K.)

2 Department of Immunology, Graduate School of Medical Science, Kyoto Prefectural University of Medicine, Kawaramachi-Hirokoji, Kamigyo-ku, Kyoto 602-8566, Japan; E-Mails: tsunao@koto.kpu-m.ac.jp (T.K.); mazda@koto.kpu-m.ac.jp (O.M.)

* Author to whom correspondence should be addressed; E-Mail: yarai89046@nike.eonet.ne.jp; Tel.: +81-75-251-5549; Fax: +81-75-251-5841.

Academic Editor: Pamela Lein

Received: 22 November 2014 / Accepted: 30 December 2014 / Published: 5 January 2015

\begin{abstract}
Hypoxia-inducible factor (HIF)-2 $\alpha$ is considered to play a major role in the progression of osteoarthritis. Recently, it was reported that pressure amplitude influences HIF-2 $\alpha$ expression in murine endothelial cells. We examined whether hydrostatic pressure is involved in expression of HIF-2 $\alpha$ in articular chondrocytes. Chondrocytes were cultured and stimulated by inflammation or hydrostatic pressure of $0,5,10$, or $50 \mathrm{MPa}$. After stimulation, heat shock protein (HSP) 70, HIF-2 $\alpha$, nuclear factor kappa B (NF- $\kappa$ B), matrix metalloproteinase (MMP)-13, MMP-3, and vascular endothelial growth factor (VEGF) gene expression were evaluated. The levels of all gene expression were increased by inflammatory stress. When chondrocytes were exposed to a hydrostatic pressure of $5 \mathrm{MPa}$, HIF-2 $\alpha$, MMP-13, and MMP-3 gene expression increased significantly although those of HSP70 and NF- $\kappa B$ were not significantly different from the control group. In contrast, HIF-2 $\alpha$ gene expression did not increase under a hydrostatic pressure of $50 \mathrm{MPa}$ although
\end{abstract}


HSP70 and NF-kB expression increased significantly compared to control. We considered that hydrostatic pressure of $5 \mathrm{MPa}$ could regulate HIF-2 $\alpha$ independent of NF- $\kappa \mathrm{B}$, because the level of HIF-2 $\alpha$ gene expression increased significantly without upregulation of NF- $\kappa B$ expression at $5 \mathrm{MPa}$. Hydrostatic pressure may influence cartilage degeneration, inducing MMP-13 and MMP-3 expression through HIF-2 $\alpha$.

Keywords: hypoxia inducible factor (HIF)-2 $\alpha$; hydrostatic pressure; chondrocytes; hypertrophic differentiation; osteoarthritis; cartilage degeneration; inflammation

\section{Introduction}

Osteoarthritis (OA) is a degenerative disease of the articular cartilage, impairing activity of daily living and quality of life due to arthralgia, limitation of the range of joint motion, and joint swelling. Mechanical loading is considered to be strongly involved in degeneration of articular cartilage. Takahashi et al. [1] showed that continuous hydrostatic pressure inhibited proteoglycan synthesis in a human chondrocyte-like cell line and Nakamura et al. [2] showed that induced apoptosis depending on pressure amplitude and/or duration in chondrocytes cultured on alginate beads. Therefore, excessive mechanical stress induces degeneration and destruction of articular cartilage, causing OA.

Hypertrophic differentiation characterized by secretion of type 10 collagen is involved in the initiation of OA. Hypertrophic differentiated chondrocytes product matrix metalloproteinase (MMP)s, resulting in progression of cartilage degeneration. Hypoxia-inducible factor (HIF)-2 $\alpha$ is a transcription factor that strongly induces type 10 collagen in chondrocytes [3,4]. Moreover, HIF-2 $\alpha$ induces expression of a wide range of factors involved in endochondral ossification, such as MMPs, vascular endothelial growth factor (VEGF), and Indian hedgehog signals, in articular chondrocytes. Recently, it was reported that pressure amplitude influences HIF-2 $\alpha$ expression in murine endothelial cells $[5,6]$. However, the influence of pressure on cartilage tissue is unknown. The present study was performed to analyze the influence of hydrostatic pressure on hypertrophic differentiation-related gene expression in cultured chondrocytes.

\section{Results}

We first examined the appropriateness of culture environments and primers by stimulating inflammation stress to cultured chondrocytes. Then, hydrostatic pressure was exposed to primary cultured chondrocytes measuring gene expression related to hypertrophic differentiation.

\subsection{Influence of Inflammation to Chondrocytes}

We measured gene expression in cultured chondrocytes after treated with interleukin (IL)-1 $\beta$. The levels of NF- $\mathrm{BB}$, HIF-2 $\alpha, \mathrm{MMP}-13, \mathrm{MMP}-3$, and VEGF gene expression were significantly increased by 5.0-, 3.7-, 26.5-, 1356-, and 1.5-fold, respectively, by inflammatory stress (Figure 1). 

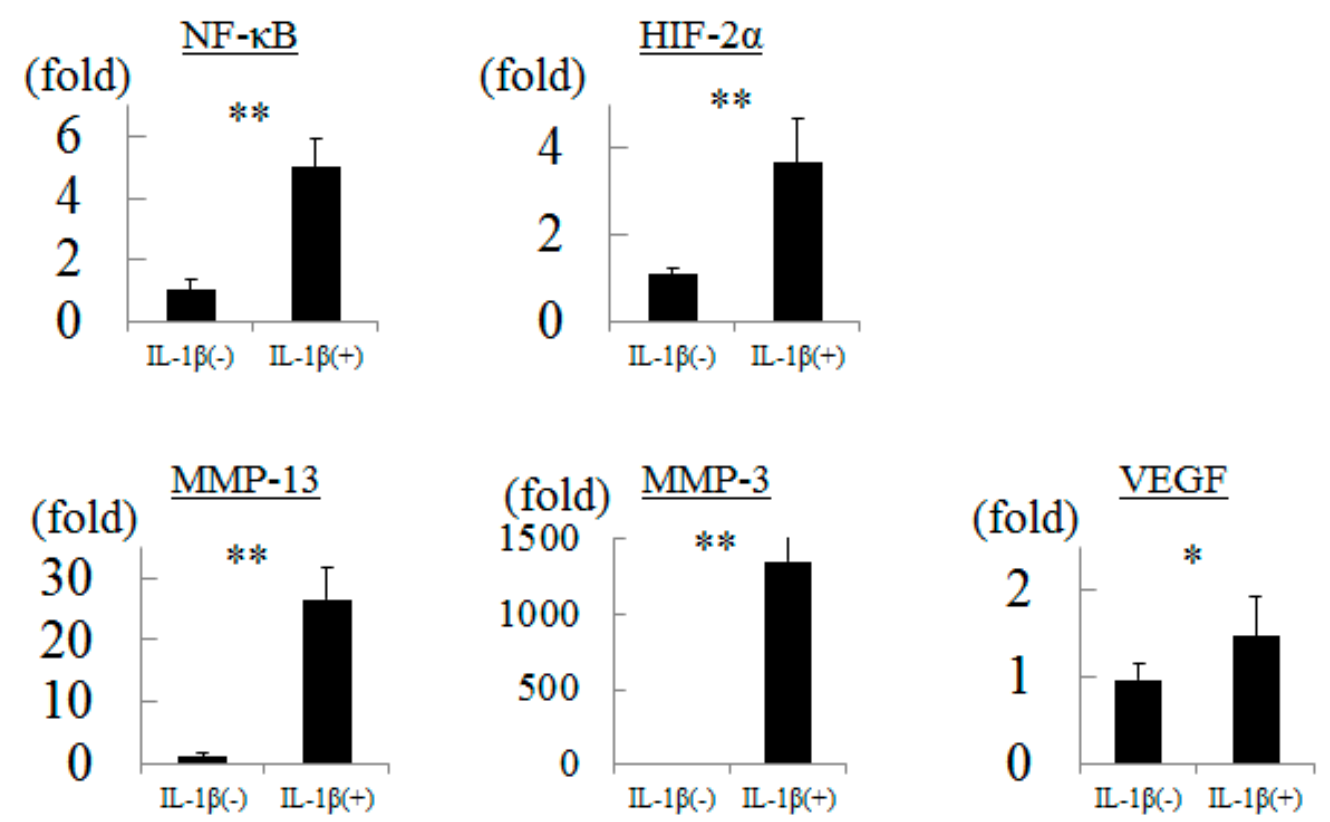

Figure 1. Gene expressions in chondrocytes after interleukin- $1 \beta$ stimulation. ${ }^{*} p<0.05,{ }^{*} p<0.01$.

\subsection{Influence of Hydrostatic Pressure to Chondrocytes}

When chondrocytes were exposed to a hydrostatic pressure of $5 \mathrm{MPa}$, HIF-2 $\alpha, \mathrm{MMP}-13$, and MMP-3 gene expression were significantly increased by 2.4-, 4.0-, and 40-fold, respectively. However, those of HSP70 and NF- $\kappa$ B were not significantly different compared to the control group (Figure 2).

In contrast, HIF- $2 \alpha$ gene expression did not increase although those of HSP70 and NF- $\kappa \mathrm{B}$ increased significantly compared to the control group under a hydrostatic pressure of $50 \mathrm{MPa}$. MMP-13 and MMP-3 gene expression in chondrocytes exposed to $50 \mathrm{MPa}$ increased significantly compared to controls, but the rate of increase was low compared to the $5 \mathrm{MPa}$ group. The level of VEGF gene expression in the $50 \mathrm{MPa}$ group was significantly increased compared to the control group (Figure 2).
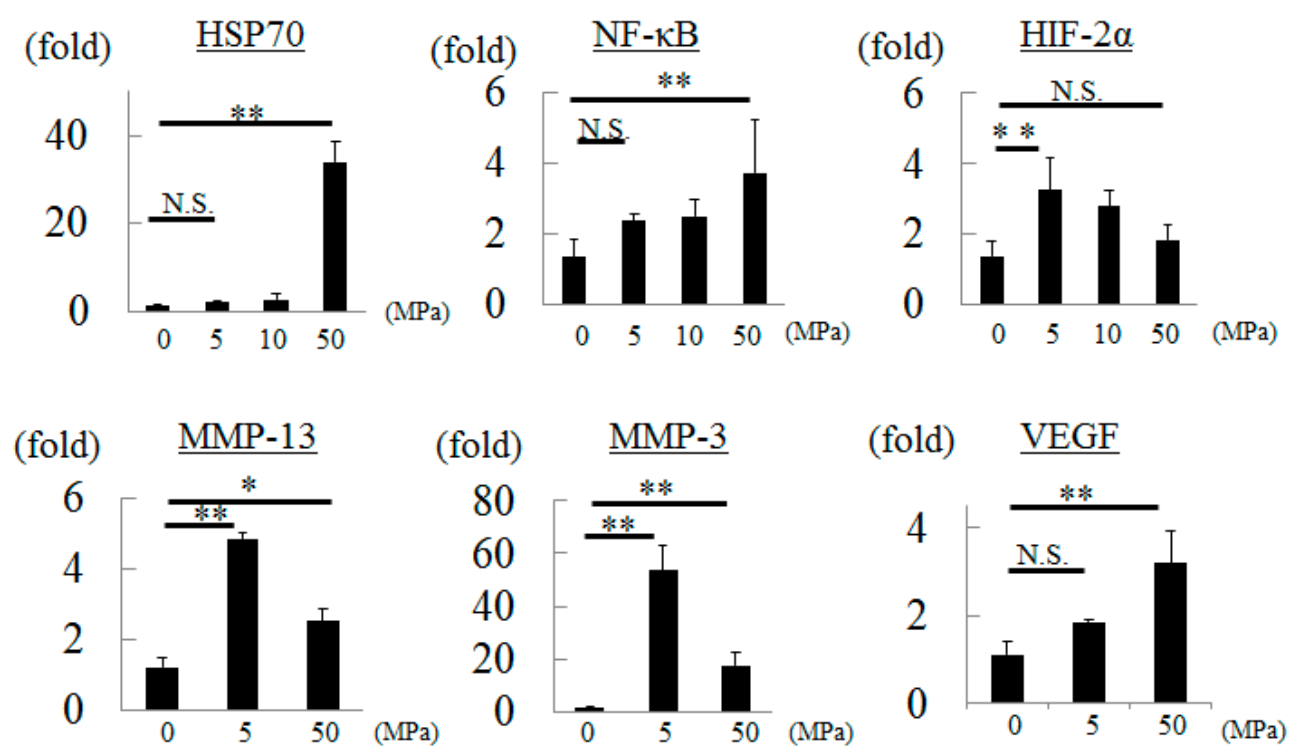

Figure 2. Gene expressions in chondrocytes after hydrostatic pressurization. ${ }^{*} p<0.05$, ** $p<0.01$, and N.S.: no significant. 


\section{Discussion}

Inflammatory stimulation enhances NF- $\kappa \mathrm{B}, \mathrm{HIF}-2 \alpha$, MMP-13, MMP-3, and VEGF gene expression in cultured chondrocytes [5]. In this study, we confirmed the appropriateness of culture environments and primers because the levels of expression of all of these genes increased as described in previous reports. HIF- $2 \alpha$ is not produced in normal articular cartilage, but is produced in human OA cartilage. It has also been clarified that surgical destabilization of the medial meniscus model using HIF-2 $\alpha$ knockout mice showed suppression of OA progression and inhibition of MMP-13 gene expression in addition to HIF-2 $\alpha$ compared to wild-type mice [3]. Therefore, HIF-2 $\alpha$ has attracted a great deal of attention as a treatment target for OA at the molecular level. Meanwhile, as it has been reported that HIF- $2 \alpha$ is upregulated by prolonged mechanical stretching in rat inferior vena cava [6] and HIF-2 $\alpha$-deficient embryonic vascular endothelial cells show downregulation of integrin [7], the expression of HIF-2 $\alpha$ may be associated with pressure or its sensor. However, there have been no previous reports regarding the relation between HIF-2 $\alpha$ and pressure in cartilage tissue. Therefore, the present study was performed to determine the relation between HIF-2 $\alpha$ and hydrostatic pressure.

Previously, we reported that continuous hydrostatic pressure induced expression of the stress protein, HSP70, in a pressure-dependent manner in a study using a chondrocyte-like cell line [1]. In this study, HSP70 gene expression did not change with exposure to a hydrostatic pressure of 5 or $10 \mathrm{MPa}$, but showed a significant increase on exposure to $50 \mathrm{MPa}$. Therefore, we performed the following experiments considering $50 \mathrm{MPa}$ as excessive hydrostatic pressure.

HSP70 has been reported to mediate NF- $\mathrm{B}$ expression in hepatocarcinoma cells [8]. Compared to the control group, there were no significant increase in HSP70 and NF- $\kappa B$ gene expression at $5 \mathrm{MPa}$, but expression of both increased significantly at $50 \mathrm{MPa}$ in the present study. Thus, we speculate that HSP70 induced by hydrostatic pressure may have a role in the expression of NF- $\mathrm{BB}$. With regard to the

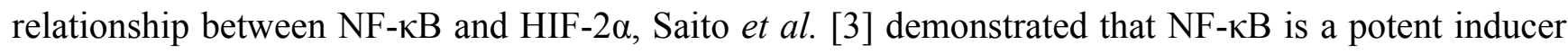
of HIF- $2 \alpha$ in HIF-2 $\alpha$ promoter assay. However, we considered the possibility that a hydrostatic pressure of $5 \mathrm{MPa}$ could actually regulate HIF-2 $\alpha$ independent of NF- $\mathrm{BB}$, because HIF-2 $\alpha$ gene expression increased significantly without upregulation of $\mathrm{NF}-\kappa \mathrm{B}$ expression at a hydrostatic pressure of $5 \mathrm{MPa}$ in the present study. Further experiments are needed to prove this. The differences between the results of the present study and those of previous reports may have been because we analyzed the influence of hydrostatic pressure alone in cultured chondrocytes. In addition, these discrepancies may also be explained by the influence of other factors produced by activation of HIF-2 $\alpha$ that may act as negative feedback, such as HIF-3 $\alpha$ [9]. MMP-13 and MMP-3 were shown to be induced by HIF-2 $\alpha[7,10]$. The levels of MMP-13 and MMP-3 gene expression also increased most at $5 \mathrm{MPa}$ compared to the controls in the present study. These observations indicated that hydrostatic pressure influences cartilage degeneration, inducing MMP-13 and MMP-3 expression through HIF-2 $\alpha$. Although it has been reported that VEGF is induced by HIF-2 $\alpha$, explaining the mechanism of regulation of VEGF is difficult because VEGF is induced by bone morphogenetic protein-2, Rho, and HIF-1 $\alpha[11,12]$. The inconsistency between expression of HIF-2 $\alpha$ and that of VEGF may be due to the influence of factors other than HIF- $2 \alpha$. 


\section{Experimental Section}

This study was conducted in accordance with the animal research guidelines of Kyoto Prefectural University of Medicine, Kyoto, Japan, and accepted by animal experiment committee as No. 24-3.

\subsection{Preparation of Chondrocytes}

Male Japanese white rabbits (Shimizu Laboratory Supplies, Kyoto, Japan) weighing $2 \mathrm{~kg}$ were killed by a lethal dose of sodium pentobarbital (Nembutal; Abbott, Abbott Park, IL, USA), and the cartilage was collected aseptically from the bilateral knee, hip, and shoulder joints. The specimens were minced into small pieces and treated with $0.015 \%$ trypsin (Gibco BRL, Gaithersburg, MD, USA) for $1 \mathrm{~h}$, followed by treatment with $0.025 \%$ collagenase (Collagenase L; Nitta Gelatin, Osaka, Japan) for 8 hours. Isolated chondrocytes were cultured for 1 week at $37{ }^{\circ} \mathrm{C}$ in $5 \% \mathrm{CO}_{2} 95 \%$ humidified air (standard conditions) in Dulbecco's modified Eagle's medium (DMEM) containing 4.5 gm/L glucose, $0.584 \mathrm{gm} / \mathrm{L}$ L-glutamine, and $0.11 \mathrm{gm} / \mathrm{L}$ sodium pyruvate (Nacalai Tesque, Kyoto, Japan), which was supplemented with 10\% fetal bovine serum (FBS) (Trace Biosciences, Melbourne, Victoria, Australia), 100 units/mL penicillin, and $100 \mu \mathrm{g} / \mathrm{mL}$ streptomycin (Gibco BRL).

\subsection{IL-1 $\beta$ Treatment}

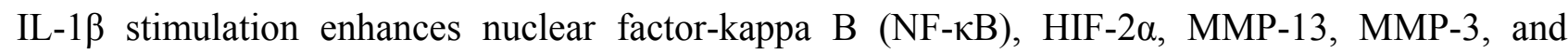
vascular endothelial growth factor (VEGF) gene expression in rabbit chondrocytes. In this study, we conducted a similar experiment to examine expression of these genes as positive controls. Chondrocytes were maintained as a monolayer, and on day 3 of culture the cells were treated with $4 \mathrm{ng} / \mathrm{mL}$ of IL-1 $\beta$ (R\&D systems, Minneapolis, MN, USA) for $24 \mathrm{~h}$.

\subsection{Pressure Application}

The cells reached confluence following approximately 1 week of culture, and were then exposed to a hydrostatic pressure of 5-50 MPa. Petri dishes were placed in a deformable polytetrafluoroethylene (Teflon) pouch that was filled with serum-free DMEM containing the same concentrations of kanamycin and L-glutamine as mentioned above and packed completely after air bubbles were removed. The pouch was then placed in a stainless steel pressurization vessel (inside diameter $65 \times 90 \mathrm{~mm}$ ), equipped with an oil pressure apparatus (type KP5B: Hikari Koatsu, Hiroshima, Japan). Kerosene was used as the pressure medium, and pressure was transmitted to the cells through the packed Teflon pouch and then the culture medium in a gas-free environment. The temperature was maintained at $37^{\circ} \mathrm{C}$ by use of a thermostat located in the pressurization vessel. We previously confirmed that there were no changes in the $\mathrm{pH}$ of the culture medium prior to and following the exposure to hydrostatic pressure. The Teflon pouch was then exposed to a hydrostatic pressure of 5,10 , or $50 \mathrm{MPa}$ for $2 \mathrm{~h}$. After depressurization, $3 \mathrm{~mL}$ of fresh serum-free DMEM was added to each Petri dish and kept at atmospheric pressure in the $5 \% \mathrm{CO}_{2}$ environment until analysis. Cells seeded in Petri dishes and placed in the same apparatus under the same conditions were used as non-pressurized controls. 


\subsection{Quantitative Real-Time Reverse Transcription-Polymerase Chain Reaction}

We studied change of HIF-2 $\alpha$ expression under excessive stress. HSP70 expression was measured as index of excessive stress. Takahashi et al. [1] have been reported gene expression of HSP70 elevation was observed at $30 \mathrm{~min}$ following the release of $50 \mathrm{MPa}$ pressure using chondrocyte-like cell line. In contrast, the elevation level was decreased at $4 \mathrm{~h}$. Thirty minutes after depressurization or $24 \mathrm{~h}$ after IL-1 $\beta$ stimulation, total RNA was extracted using Sepasol-RNA super II (Nacalai Tesque) and subjected to quantitative real-time reverse transcription-polymerase chain reaction (RT-PCR) using a Biosystems 7300 (Applied Biosystems, Foster City, CA, USA). RT-PCR was performed for HIF-2 $\alpha$, for NF- $\kappa \mathrm{B}$, for MMP-3, for MMP-13, and for VEGF with gene-specific primers and TaqMan probe (Table 1). Moreover, HSP70 gene expression was used as a marker for evaluation of hydrostatic pressure exposure of cultured chondrocytes. Quantitative RT-PCR was performed using a Biosystems 7300 with TaqMan Assay-on Demand gene expression primer/probe sets (Applied Biosystems) for HSP70 (Assay ID; AJ297379). To quantify changes in gene expression, the comparative Ct method was used to calculate the relative fold changes normalized relative to ribosomal RNA. Results are shown as the means of three samples, with each sample assayed in duplicate.

Table 1. Primers used for real-time RT-PCR.

\begin{tabular}{|c|c|c|}
\hline Gene & Primer Sequence & Probe No. \\
\hline $\mathrm{HIF}-2 \alpha$ & $\begin{array}{l}\text { Forward: agcttcctgcggacacac } \\
\text { Reverse: cctcggcttcagactcattt }\end{array}$ & 73 \\
\hline $\mathrm{NF}-\kappa \mathrm{B}$ & $\begin{array}{l}\text { Forward: aagaccaggtttccgaggac } \\
\text { Reverse: tgcaccatgaggaaagaagtt }\end{array}$ & 133 \\
\hline MMP-3 & $\begin{array}{l}\text { Forward: tggacctggaaatgttttgg } \\
\text { Reverse: atcaaagtgggcatctccat }\end{array}$ & 72 \\
\hline MMP-13 & $\begin{array}{c}\text { Forward: cctcttcttctccggaaacc } \\
\text { Reverse: ggtagtcttggtccatggtatga }\end{array}$ & 50 \\
\hline VEGF & $\begin{array}{l}\text { Forward: ctacctccaccatgccaagt } \\
\text { Reverse: ccacttcgtggggtttattg }\end{array}$ & 29 \\
\hline $18 \mathrm{~S}$ ribosomal RNA & $\begin{array}{l}\text { Forward: atgagtccactttaaatcctttaacga } \\
\text { Reverse: ctttaatatacgctattggagctggaa }\end{array}$ & - \\
\hline
\end{tabular}

\subsection{Statistical Analysis}

All data are presented as the mean \pm standard deviation (SD). The data were analyzed by analysis of variance (ANOVA), and post hoc testing was performed using the Tukey-Kramer test.

\section{Conclusions}

In conclusion, we showed that expression of HIF-2 $\alpha$ in cultured chondrocytes is induced independent of NF- $\mathrm{KB}$ expression under a hydrostatic pressure of $5 \mathrm{MPa}$. These results may help in elucidation of the pathology of OA because HIF- $2 \alpha$ and MMPs, acting downstream of HIF-2 $\alpha$, are involved in initiation and progression of OA. 


\section{Acknowledgments}

This work was supported by grants from KAKENHI (Nos. 24390356 and 26462312).

\section{Author Contributions}

Conceived and designed the experiments: Hiroaki Inoue, Yuji Arai, Ryu Terauchi, Kuniaki Honjo, Shuji Nakagawa, Shinji Tsuchida; Performed the experiments: Hiroaki Inoue, Tsunao Kishida, Tomohiro Matsuki; Analyzed the data: Hiroaki Inoue, Yuji Arai, Shuji Nakagawa; Wrote the manuscript: Hiroaki Inoue, Yuji Arai, Keiichirou Ueshima, Hiroyoshi Fujiwara, Osam Mazda, Toshikazu Kubo.

\section{Conflicts of Interest}

The authors declare no conflict of interest.

\section{References}

1. Takahashi, K.; Kubo, T.; Kobayashi, K.; Imanishi, J.; Takigawa, M.; Arai, Y.; Hirasawa, Y. Hydrostatic pressure influences mRNA expression of transforming growth factor- $\beta 1$ and heat shock protein 70 in chondrocyte-like cell line. J. Orthop. Res. 1997, 15, 150-158.

2. Nakamura, S.; Arai, Y.; Takahashi, K.A.; Terauchi, R.; Ohashi, S.; Mazda, O.; Imanishi, J.; Inoue, A.; Tonomura, H.; Kubo, T. Hydrostatic pressure induces apoptosis of chondrocytes cultured in alginate beads. J. Orthop. Res. 2006, 24, 733-739.

3. Saito, T.; Fukai, A.; Mabuchi, A.; Ikeda, T.; Yano, F.; Ohba, S.; Nishida, N.; Akune, T.; Yoshimura, N.; Nakagawa, T.; et al. Transcriptional regulation of endochondral ossification by HIF-2 $\alpha$ during skeletal growth and osteoarthritis development. Nat. Med. 2010, 16, 678-686.

4. Yang, S.; Kim, J.; Ryu, J.H.; Oh, H.; Chun, C.H.; Kim, B.J.; Min, B.H.; Chun, J.S. Hypoxia-inducible factor-2 $\alpha$ is a catabolic regulator of osteoarthritic cartilage destruction. Nat. Med. 2010, 16, 687-693.

5. Ryu, J.H.; Yang, S.; Shin, Y.; Rhee, J.; Chun, C.H.; Chun, J.S. Interleukin-6 plays an essential role in hypoxia-inducible factor $2 \alpha$-induced experimental osteoarthritic cartilage destruction in mice. Arthritis Rheumatol. 2011, 63, 2732-2743.

6. Lim, C.S.; Qiao, X.; Reslan, O.M.; Xia, Y.; Raffetto, J.D.; Paleolog, E.; Davies, A.H.; Khalil, R.A. Prolonged mechanical stretch is associated with upregulation of hypoxia-inducible factors and reduced contraction in rat inferior vena cava. J. Vasc. Surg. 2011, 53, 764-773.

7. Skuli, N.; Liu, L.; Runge, A.; Wang, T.; Yuan, L.; Patel, S.; Iruela-Arispe, L.; Simon, M.C.; Keith, B. Endothelial deletion of hypoxia-inducible factor-2 $\alpha$ (HIF-2 $\alpha$ ) alters vascular function and tumor angiogenesis. Blood 2009, 114, 469-477.

8. Gong, W.; Wang, Z.Y.; Chen, G.X.; Liu, Y.Q.; Gu, X.Y.; Liu, W.W. Invasion potential of H22 hepatocarcinoma cells is increased by HMGB1-induced tumor NF- $\kappa \mathrm{B}$ signaling via initiation of HSP70. Oncol. Rep. 2013, 30, 1249-1256. 
9. Yamashita, T.; Ohneda, O.; Nagano, M.; Iemitsu, M.; Makino, Y.; Tanaka, H.; Miyauchi, T.; Goto, K.; Ohneda, K.; Fujii-Kuriyama, Y.; et al. Abnormal heart development and lung remodeling in mice lacking the hypoxia-inducible factor-related basic helix-loop-helix PAS protein NEPAS. Mol. Cell Biol. 2008, 28, 1285-1297.

10. Hirata, M.; Kugimiya, F.; Fukai, A.; Saito, T.; Yano, F.; Ikeda, T.; Mabuchi, A.; Sapkota, B.R.; Akune, T.; Nishida, N.; et al. C/EBP $\beta$ and RUNX2 cooperate to degrade cartilage with MMP-13 as the target and HIF-2 $\alpha$ as the inducer in chondrocytes. Hum. Mol. Genet. 2012, 21, 1111-1123.

11. Sun, H.; Breslin, J.W.; Zhu, J.; Yuan, S.Y.; Wu, M.H. Rho and ROCK signaling in VEGF-induced microvascular endothelial hyperpermeability. Microcirculation 2006, 13, 237-247.

12. Kondo, A.; Otsuka, T.; Kuroyanagi, G.; Yamamoto, N.; Matsushima-Nishiwaki, R.; Mizutani, J.; Kozawa, O.; Tokuda, H. Resveratrol inhibits BMP-4-stimulated VEGF synthesis in osteoblasts: suppression of S6 kinase. Int. J. Mol. Med. 2014, 33, 1013-1018.

(C) 2015 by the authors; licensee MDPI, Basel, Switzerland. This article is an open access article distributed under the terms and conditions of the Creative Commons Attribution license (http://creativecommons.org/licenses/by/4.0/). 